\title{
Numerical and Experimental Study of Interference Based Micromachining of Stainless Steel
}

\author{
Avinash Parashar*, Jasjit Singh Mann, Ankur Shah, NR Sivakumar \\ Department of Mechanical and Industrial Engineering, EV13-310, Concordia University, \\ 1515, St. Catherine West, Montréal, Quebec, Canada H3G 2W1 \\ E-mail:parasharavinash@yahoo.com
}

\begin{abstract}
An interference based nanosecond laser micromachining method for stainless steel has been investigated. Predictive mathematical modeling was carried out using MATLAB to identify the optimum laser parameters for the micromachining of stainless steel. Experiments on machining of stainless steel sample plates were then performed with a nanosecond pulse length laser beam of central wavelength $1064 \mathrm{~nm}$. Marking of the material was achieved at different power levels, pulse repetition rates and machining times. The modeling and experimental results were compared, with proper justification.

DOI: $10.2961 /$ jlmn.2009.02.0009
\end{abstract}

Keywords: Interference, Wollaston prism

\section{Introduction}

Short pulsed laser processing is an enabling technology facilitating component miniaturization and improved performance characteristics. Among pulsed lasers, short-pulsed lasers are of the current research interest [1]. Micro- and nanosecond lasers have much higher pulse energy compared to picosecond (ps) and femtosecond (fs) types, which makes long pulse ablation more efficient in removal of material from a surface [2]. Ultrafast lasers are ideal for development purposes and may be considered for certain specialist applications production, where they show unique benefits, however nanosecond laser technology is mature and is widely adopted in the industry for micromachining and marking applications [3]. Ink-marking, mechanical engraving and electro-chemical are example of some of the marking methods but laser marking finds a place when reliable, easily customized and indelible marking is desired [4-7]. Interference based micromachining also have many applications in photonics but predominant application of lye's in the field of marking [8].

J.Qi et al studied stainless steel marking with $\mathrm{Nd}$ :YAG laser with respect to depth, width and contrast of the machining [9]. Experimental and theoretical analysis of micromachining of stainless steel was described by A.A.I. Khalil [10]. The influence of focal conditions on spatial profiles of plasma, emission intensity and averaged ablation rate of stainless steel samples as a function of laser energy was studied and discussed in detail by L. M. Cabalín [11].though several attempts has been made for the laser based marking of metals, interference based marking has been not studied so far in depth. S.F.Ho and B.K.A. Ngoi published their work on modeling of interference intensity of the central fringe for sub micron drilling. But still the effect of pulse energy along with the rep rate for micromachining was not studied in depth with interference principle and also the affect of these parameters on all the fringes along with central fringe was not addressed in the research paper [11].
$\mathrm{Nd}: \mathrm{YVO}_{4}$ laser based micromachining of stainless steel is studied in this research paper. Our principal objective is to provide a better understanding of stainless steel machining based on interference principle. Predictive modeling of interference based micromachining has been done with different laser parameters. Experiments were done further to justify the results obtained with mathematical modeling.

\section{Experimental Setup}

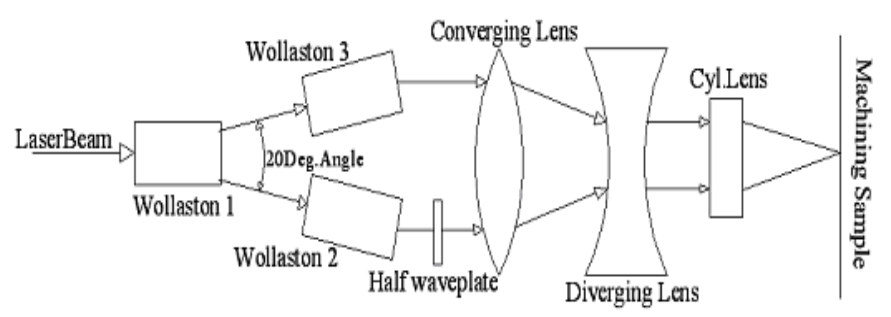

Figure 1 Micro-machining setup

The experimental setup was designed and modified with the ability to form fringes at the focal point from research paper [8]. A pulsed laser beam of 14 ns pulse width from $\mathrm{Nd}: \mathrm{YVO}_{4}$ laser is spatially filtered and the diameter of the beam is expanded to $4.8 \mathrm{~mm}$ from $0.8 \mathrm{~mm}$ diameter. The beam coming out from the pulsed laser is then circularly polarized using a quarter waveplate. The circularly polarized laser beam is made to pass through a $20^{\circ}$ first Wollaston prism. The output of the first Wollaston prism is therefore two orthogonally polarized beams with an angle of $20^{\circ}$ between them. In order to make these two beams parallel so that it can be focused at a common point using single lens, each of the two orthogonally polarized beams is than made to pass through two separate $20^{\circ}$ Wollaston prisms as shown in the figure1. It is important to note that, in order to get interference from the two beams, the polarization of one of the beams needs to be changed while maintaining the polarization of the other. The polarization of one beam can be changed by using one half waveplate on any one of the parallel beams. In the previously proposed setup [8] beam spacing between the two parallel beams can only be changed by displacing the 
Wollaston prisms 2 and 3 which is a tedious process, whereas in the current proposed setup the beam spacing can be easily managed with different set of diverging lenses and the variation in the beam diameter due to this arrangement can be easily managed with telescopic arrangements.

\section{Results and Discussions}

Experiments on laser ablation were performed using a range of pulse energies, pulse repetition rates and number of shots. The stainless steel sample was kept on a 3D stage exactly at the focal of the focusing lens. Due to the occurrence of interference between the two superimposed focusing beams at the focal point of the cylindrical lens $(100 \mathrm{~mm})$, fringes were machined on the stainless steel sample surface. Cylindrical lens was used for the experiments, keeping in view that predominant application of metal micromachining is in marking therefore, longer lengths can be marked in a single shot. Machined spots of stainless steel were then studied with the help of scanning electron microscope (SEM) and optical microscope.

\subsection{Effect of pulse energy on micromachining}

The principle of interference was used for the micromachining of stainless steel. A Gaussian intensity distribution with interference in space for focused spot with cylindrical lens is given by I as discuss in equation (1):

$$
I=\left(I_{1}+I_{2}+2 \sqrt{ } I_{1} I_{2} \operatorname{Cos} \delta\right) \exp -\left(x^{2} / w_{o}{ }^{2}+y^{2} / w_{b}{ }^{2}\right)
$$

Where $I_{1}$ and $I_{2}$ is the beam intensity in the two parallel beams respectively, $\delta$ is the phase difference between the interfering beams, $\mathrm{w}_{\mathrm{o}}$ is the waist radius of the Gaussian beam , $\mathrm{w}_{\mathrm{b}}$ is the initial radius of unfocused laser beam , $\mathrm{x}$ and $\mathrm{y}$ are the coordinates, where intensity $\mathrm{I}$ is required. Pulse energy of the laser can be varied either by changing the average power or by changing the repetition rate (rep rate) of the laser. To identify the optimum laser parameters, theoretical modeling has been done to predict the behaviour of pulse energy on micromachining as shown in figure 2 and 3 . In figure 2 pulse energy of single pulse is varied with average power of the laser whereas in figure 3 the pulse energy is varied with respect to rep rate of the laser.

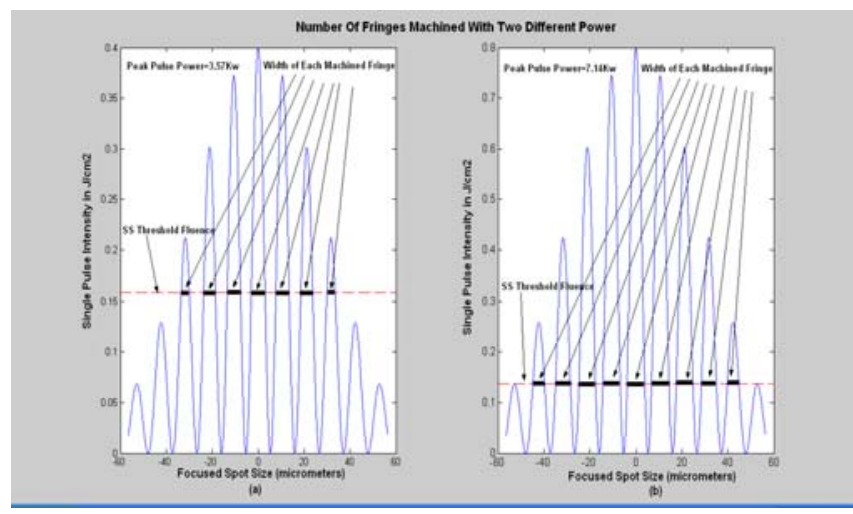

Figure 2 (a) Fringes above threshold at 1watt of average laser power (b) Fringes above threshold at 2 watt of average laser power
In figures $2 \& 3 x$ axis shows the focused width of the spot with a cylindrical lens of $100 \mathrm{~mm}(f)$ and beam diameter of $2.3 \mathrm{~mm}$ while the $y$ axis represents pulse energy distribution according to equation (1). As the cylindrical lens only focuses in one axis, width of the laser spot is calculated on the basis of $86.5 \%$ of energy inside the spot which is given by equation (2) whereas the length of the spot remains the same as the diameter of the laser beam.

Focused Spot Width $=2.44($ wavelength $)\left(f / 2 w_{b}\right)$

From figure 2 (a) and (b) it can be theoretically predicted that number of fringes machined on stainless steel depends on pulse energy. With increase in pulse energy, intensity profile within the focused spot changes and now more number of fringes has intensity above the threshold of stainless steel. Fringes having fluence above the threshold of stainless steel will take place in micromachining, whereas fringes in which fluence is lower than the threshold will not take place in micro machining. Similar modeling was also carried out with rep rates of $20 \mathrm{kHz}$ and $30 \mathrm{kHz}$ with constant average power as shown in figure 3 , laser beam diameter and focusing lens was kept constant for both the modeling.

The relationship between energy of pulse (Joules), Rep rate $(\mathrm{kHz})$ and average power (watt) is given by equation (3).

\section{Energy per Pulse = Average Power/Repetition Rate}

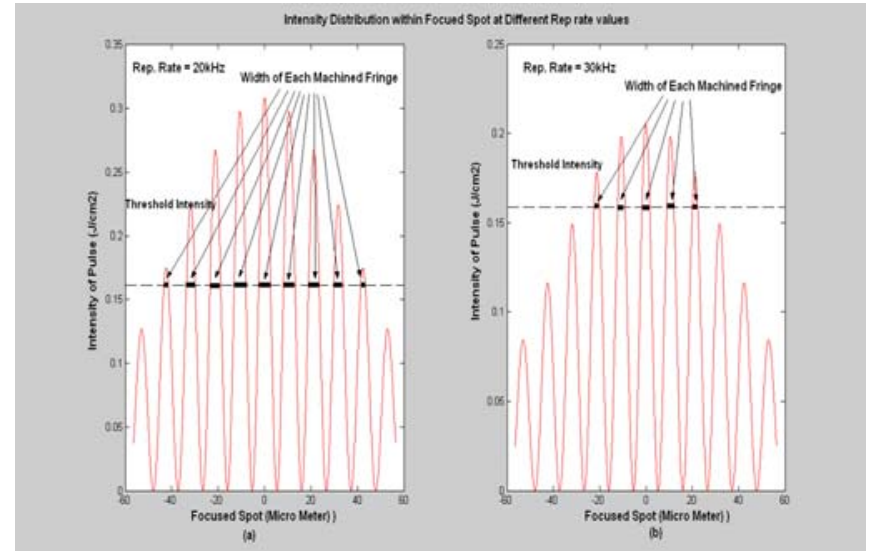

Figure 3 (a) Fringes above threshold at $20 \mathrm{kHz}$ of rep rate (b) Fringes above threshold at $30 \mathrm{kHz}$ of rep rate

From the mathematical calculations it can be said that less number of fringes have fluence above threshold of stainless steel at higher rep rate values in comparison to number of fringes above threshold fluence at lower rep rate values.

Experiments were done with multiple pulse energies, beam diameter of $2.3 \mathrm{~mm}$ with a cylindrical lens of focal length $100 \mathrm{~mm}$ and the results were plotted in figure 4 . 


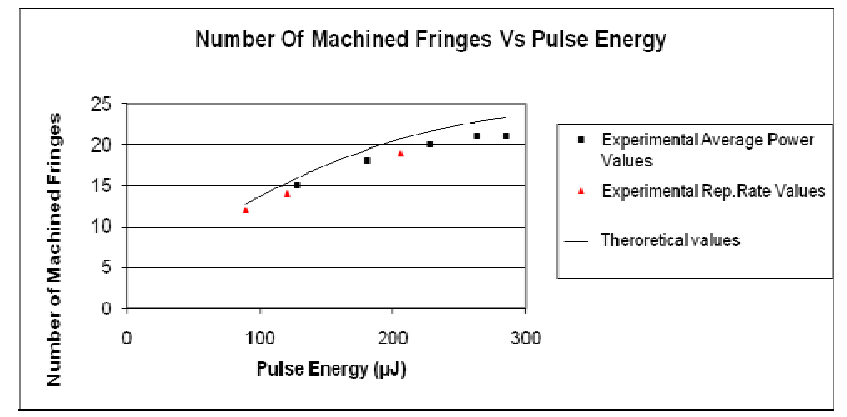

Figure 4 Fringes machined with different pulse energies

From the plotted experimental values in figure 4 it can be concluded that initially the slope of the curve is steep, therefore a change in pulse energy from $84 \mu \mathrm{J}$ to 196 $\mu \mathrm{J}$ will significantly increase the number of machined fringes. Increases in the machined fringes were not significant at higher values of pulse energy as indicated in figure 4. This indicates that most of the heat supplied at higher pulse energies is either conducted through the material or convected to the surrounding medium at high peak pulse energies, therefore only increases the heat affected zone without significantly increasing the quantity of machining. As conduction and convection losses with exact absorption of wavelength on stainless steel cannot be exactly predicted which accounts for the deviation in experimental and theoretical values. At higher pulse energies losses will increase which attributes the high variation in theoretical and experimental values.

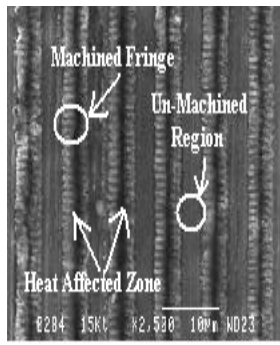

(a)

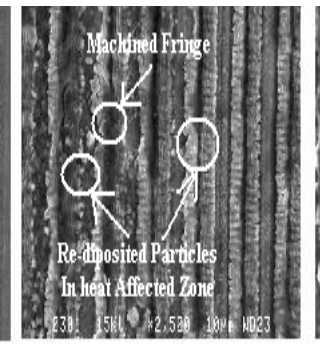

(b)

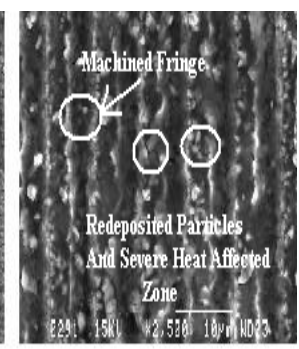

(c)
Figure 5 Marking done at a pulse energy of (a) $129 \mu \mathrm{J}$ (b) $165 \mu \mathrm{J}$ (c) $227 \mu \mathrm{J}$

The quality of the machining was also studied with respect to pulse energy. Stainless steel samples were machined with pulse energies of $129 \mu \mathrm{J}, 165 \mu \mathrm{J}$ and $227 \mu \mathrm{J}$ at a constant repetition rate of $20 \mathrm{kHz}$. SEM images of the three marked regions on stainless steel are shown in figure 5. From figure 5 it can be concluded that marking done at $129 \mu \mathrm{J}$ of pulse energy (figure $5 \mathrm{a}$ ) has less heat affected zone in comparison to marking done at $165 \mu \mathrm{J}$ and $227 \mu \mathrm{J}$ (figure $5 \mathrm{~b} \&$ figure 5c). At higher pulse energies conduction of laser energy into the sample material was more, which increases the heat affected region and also redeposition of machined particles taken place at such high pulse energies as shown in figure 5(c), ultimately reducing the quality of machining.

The quality of the machining was also studied with respect to different rep rates of pulse laser as shown in figure 6. From the SEM images in figure 6, it can be said that quality of machining improves, if done at higher rep rate values in comparison to marking done at lower rep rates. Machining done at lower rep rate values of $20 \mathrm{kHz}$ have high heat affected zone in comparison to machining done at higher rep rate of $30 \mathrm{kHz}$. whereas machining done at $40 \mathrm{kHz}$ rep rate has best machining quality in terms of negligible amount of heat affected zone and re-deposited material. High quality of machining at higher rep rates can be attributed to low pulse energy at high rep rates which has been explained earlier with the modeling.

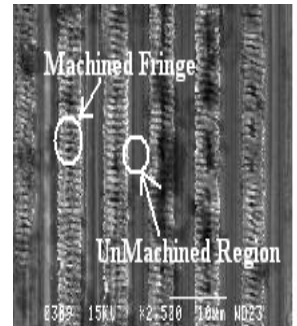

(a)

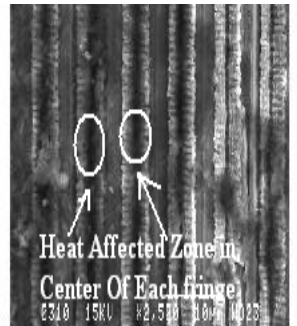

(b)

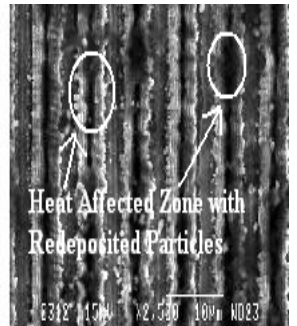

(c)
Figure 6 Marking done at (a) $40 \mathrm{kHz}$, (b) $30 \mathrm{kHz}$ (c) $20 \mathrm{kHz}$

\subsection{Machining with different number of shots}

In addition to variation of pulse energy, number of shots also plays an important role in micromachining which has a significant effect on ablated depth. To study this experiment were done with different number of pulses hitting the stainless steel sample. Depth of machining was calculated for each machined spot with respect to different number of shots. Experimental results are shown in figure 7 for different depths obtained with increase in number of shots. Number of shots for the experiments was controlled with the scanning speed of the stainless steel sample on the mounting stage. With increase in the scanning speed less number of pulses will hit the same machining spot; in this manner number of shots could be controlled during the experiments.

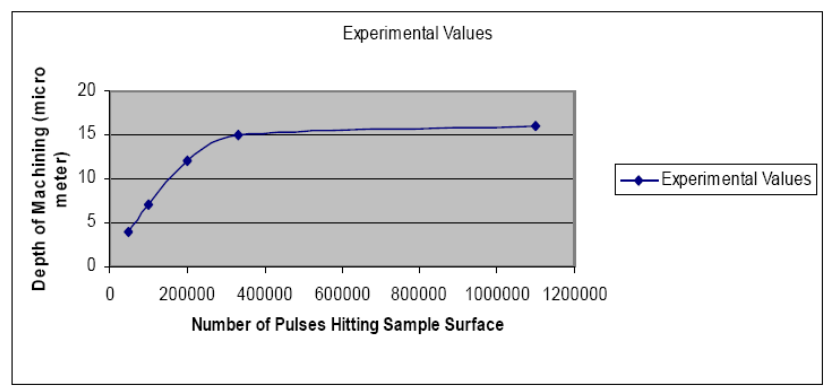

Figure 7 Experimental details of marking done at $210 \mu \mathrm{J}$ of pulse energy at different rep rates

Depth of machining increases with increase in the number of pulses hitting the same region as shown in figure 7 , but after certain number of pulses a saturation limit was reached and this limit increase in depth with increase in number of shot was found to be negligible. The machining limit depends on the material properties, with increase in number of shots temperature of material increases which will ultimately increase the conduction losses as well as convection current losses with the surrounding medium and 
will reduce the machining. Machining will also be reduced due to formation of molten metal pool at the bottom of the machined spot which increases the pulse energy losses [12].

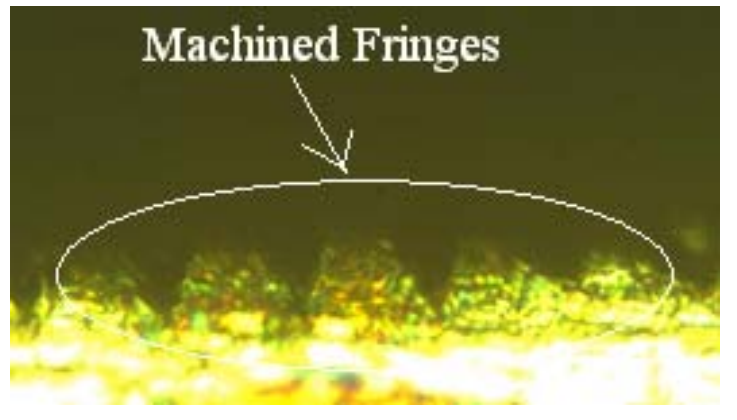

Figure 8 Machined fringes with $189 \mu \mathrm{J}$ pulse energy at $20 \mathrm{kHz}$ rep rate

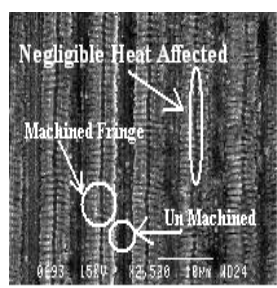

(a)

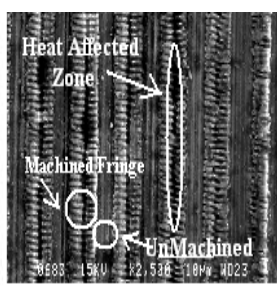

(b)

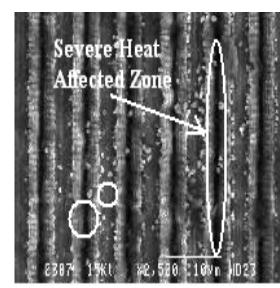

(c)
Figure 9 Marking done with (a) 100,000 shots (b) 200,000 shots (c) 500,000 shots

Machining done with lesser number of pulses has well defined depth for each fringe, whereas quality of the machined patterns reduces significantly with higher number of pulses due to high heat affected region and material redeposition as shown in figure 9 . The reduction in quality of the machining is attributed to the more conduction of heat in the machining region due to increased number of shots above the saturation limit. From the images in figure 9 it can be said that machining done with 100,000 shots have negligible amount of heat affected zone in the center of each fringe whereas no material re-deposited, on the other hand machining done with 200,000 shots have little heat affected zone in the center of some of the fringes and also no re-deposition of material. In contrast to the machining done with 100,000 and 200,000 shots, machining done with 500,000 shots have high heat affected zone and significant amount of material re-deposition.

\section{Conclusions}

Numerical and experimental analysis of interference based micromachining of stainless steel has been done. An experimental setup has been described for creating fringes at the focal point using a single focusing lens. Mathematical modeling along with experiments was done to identify the optimal laser parameters for maintaining the equality and quantity in micromachining of stainless steel. Experiments results were found to in good match with the theoretical calculations.

\section{REFERENCES}

[1] Meijer, J., Du, K., Gillner, A., Hoffmann, D., Kovalenko, V.S., Masuzawa, T., Ostendorf, A., Poprawe, R., and Schulz, W. (2002), "Laser machining by short and ultrashort pulses, state of the art and new opportunities in the age of the photons," Annals of the CIRP, 51/2, 531-652.

[2] Ion, John C, "Laser Processing of Engineering Materials"Elsevier/Butterworth-Heinemann (Boston) 2005

[3] M. R. H. Knowles, G. Rutterford \& D. Karnakis, A. Ferguson "Micro-machining of metals, ceramics and polymers using nanosecond lasers" J. Adv Manuf Technol (2007) 33:95-102.

[4] H. Jundt, J. Junghans, "Microscopic material interactions by laser engraving", Proc. SPIE 744 (1987) 147-155.

[5] Isamu Miyamoto, Koji Sugioka, Thomas W. Sigmon, "Laser Precision Micro fabrication", Proceedings of SPIE -- Volume 4088 First International Symposium (2000), pp. 363-366

[6] Friedrich H. Dausinger, Vitali I. Konov, Vladimir Y. Baranov, Vladislav Y. Panchenko, Laser Processing of Advanced Materials and Laser Micro technologies, Editors Proceedings of SPIE -- Volume 5121 September 2003, pp. 157-163

[7] Th. Dumonta, T. Lipperta, A. Wokauna, P. Leyvraz "Laser writing of 2D data matrices in glass"J. Thin Solid Films 453 -454 (2004) 42-45

[8] Bo Tan1, Narayanswamy R Sivakumar2 and Krishnan Venkatakrishnan1 "Direct grating writing using femtosecond laser interference fringes formed at the focal point" J. Opt. A: Pure Appl. Opt. 7 (2005) pp(169-174)

[9] J. Qi ,K.L.Wang and Y.M.Zhu; "A study on the laser marking process of stainless steel"; Journal of Materials Processing Technology 139 (2003) 273276.

[10] A.A.I. Khalil and N. Sreenivasan, "Study of experimental and numerical simulation of laser ablation in stainless steel" Laser Phys. Lett. 2, No. 9, 445-451 (2005)

[11] S.F. Ho and B.K.A.Ngoi "Sub-microdrilling with ultrafast pulse laser interference” Appl. Phys. B 79, 99-102 (2004)

[12] Basem F, Yousef Ec and George K. Knopf; "Neural network modeling and analysis of the material removal process during laser machining" J Adv Manuf Technol (2003) 22: pp(41-53)

(Received: April 7, 2009, Accepted: August 5, 2009) 